\title{
MEMS based hair flow-sensors as model systems for acoustic perception studies
}

\author{
Gijs J.M. Krijnen, M. Dijkstra, J.J. van Baar, \\ J.H. de Boer, T.S.J. Lammerink, R. Wiegerink. \\ Transducers Science and Technology group \\ $\mathrm{MESA}^{+}$Research Institute, University of Twente \\ P.O. Box 217, 7500AE, Enschede, The Netherlands. \\ Email: g.j.m.krijnen@el.utwente.nl
}

\begin{abstract}
Arrays of MEMS fabricated flow sensors inspired by the acoustic flow-sensitive hairs found on the cerci of crickets, have been designed, fabricated and characterized. The hairs consist of up to $1 \mathrm{~mm}$ long SU-8 structures mounted on suspended membranes with normal translational and rotational degrees of freedom. Electrodes on the membrane and on the substrate form variable capacitors allowing for capacitive read-out. Capacitance versus voltage, frequency dependency and directional sensitivity measurements have been successfully carried out on fabricated sensor arrays, showing the viability of the concept.

The sensors form a model-system allowing for investigations on sensory acoustics by their arrayed nature, their adaptivity via electrostatic interaction (frequency tuning and parametric amplification) and their susceptibility to noise (stochastic resonance)
\end{abstract}

\section{Introduction}

Sensory systems in biology are among the most sensitive and evolved known to man. In comparing biological and engineered systems for similar functions one often finds striking differences in implementation; for example when taking a look at auditory sensing. 1) In biological systems sensing elements are based on the flow-sensitivity of large arrays of parallel operating mechano-sensors (hairs, cilia). 2) Biological systems rely heavily on mechanical filtering and amplification. And 3) noise may play a beneficial role when perceiving signals near the noise limits. Engineered systems for acoustic sensing on the other hand are based on pressure measurements using single moving structures (e.g. membranes as in microphones), perform filtering and amplification in the electronic domain (in a sequential manner), and generally see their usable dynamic sensing range being limited by noise. An example of mechanical filtering in biology is found in the auditory system of mammals where tapered "sound-board" like resonator structures (basilar membrane) with complex interacting inner- and outer- hair-cells perform distributed filtering, amplification and adaptation in the mechanical domain [1]. At the same time parallelism helps to overcome constraints of bandwidth of the neural systems and provides robustness and gradual decline. Examples of how biological systems benefit from noise are seen in the form of stochastic resonance and amplification [2] as observed in crickets [3] and crayfish [4]).

Despite the advancement of mankind in many areas of technology it is still challenging for engineered systems to compete with biological systems. For example: the auditory capabilities of bats to perceive their environment, locate prey and to navigate at high velocities through complex surrounding (e.g. with leafed brushes and trees) has no manmade equivalent. Likewise the sensitivity of hair-based auditory mechano-sensors, found on insects [5], to precieve acoustic signals at thermal noise levels is astounding. It is this kind of performance that raises interest in biological systems with the purpose to improve engineered systems.

There seems to be a favourable size match between the primary sensing parts (e.g. mechano-sensing hairs found on cricket cerci with lengths roughly between 100 - $1000 \mu \mathrm{m}$ ) and what can be made by a technology generally denoted by "Micro Electro Mechanical Systems" (MEMS). It allows, in principle, for bio-mimicking of biological sensory systems such as the flow sensitive hairs of cricket, the tactile hair sensors on spiders [6], the lateral line sense organs of fish [7] and the mammalian cochlea [1]. 


\section{Cricket sensory hairs}

Crickets have achieved acoustic sensing in one form by evolving sensitive mechanoreceptive sensory hairs. These so called filiform hairs are highly perceptive to low-frequency sound with energy sensitivities below thermal threshold [5].

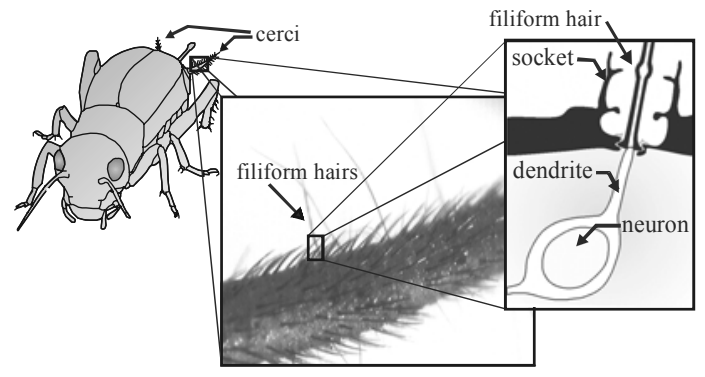

Figure 1. Filiform hairs on the cerci of crickets [8].

The sensory hairs of the cricket are situated on the back of the cricket's body on appendices called cerci. The hairs vary in length up to around $1 \mathrm{~mm}$, with a bimodal distribution with concentrations around $150 \mu \mathrm{m}$ and 750 $\mu \mathrm{m}$ [9]. Each hair is lodged in a socket, guiding the hair to move in a preferred direction. The hair is held in its socket by an elastic material surrounding the base. Airflow causes a neuron to fire, by rotation of the hair base (figure 1). The cricket is able to pinpoint lowfrequency sound from any given direction using the combined neural information of all sensory hairs [10].

The mechanics of the sensory hairs of crickets has been modelled extensively [e.g. 11, 12]. Filiform hairs can be described as an inverted pendulum, a second order mechanical system, which is fully determined by the spring constant $\mathrm{K}_{\vartheta}$, the moment of inertia $\mathrm{J}$ and the torsional resistance $\mathrm{R}$. Typical values for cricket filiform hairs with a length of $200 \mu \mathrm{m}$ are: $\mathrm{K}_{9} \approx 1012 \mathrm{Nm} \cdot \mathrm{rad} 1$, $\mathrm{J} \approx 5 \cdot 1021 \mathrm{~kg} \cdot \mathrm{m} 2$ and $\mathrm{R} \approx 1016 \mathrm{Nms} \cdot \mathrm{rad} 1$ [11]. This gives a critically damped system with a damping coefficient of 0.7. Resonance frequencies of filiform hairs are in the range of 85 to $500 \mathrm{~Hz}$. Impedance matching between the torsional resistance $\mathrm{R}$ and the drag resistance on the hair allows for maximum energy transfer, hence optimised sensitivity of the sensory hairs [11].

The filiform hairs are deflected by drag-forces on the hair shaft, due to particle velocity surrounding the cercus. The frequency dependent velocity profile above a flat surface is given by $[11,12]$ :

$$
\begin{gathered}
v_{y}(x, t)=V_{0} \cdot \sin (\omega t)-V_{0} \cdot e^{-\beta x} \cdot \sin (\omega t-\beta x) \\
\beta=\sqrt{\frac{\omega}{2 \cdot v}}
\end{gathered}
$$

with $v$ being the kinematic viscosity, $\mathrm{x}$ the distance from the surface and $\omega$ the angular frequency of the harmonically oscillating flow. The boundary layer thickness depends on $\beta^{-1}$, being larger at lower frequencies.
The total torque on the filiform hairs can be calculated by integrating the drag-moment along the hair. The drag-forces can be determined by Oseen's drag-force approximation [10]:

$$
\begin{aligned}
& F_{\text {drag }}(x, t)=8 \pi \mu \cdot \frac{v_{y}(x, t)}{2 \cdot S(x, t)+1} \\
& S(x, t)=\ln \left(\frac{8}{\operatorname{Re}(x, t)}\right)-\gamma \quad \gamma=0.577 \\
& \operatorname{Re}(x, t)=\left|\frac{2 a \cdot v_{y}(x, t)}{v}\right|
\end{aligned}
$$

with $\mu$ being the dynamic viscosity, a the radius of the hair, and $\gamma$ being Euler's constant. Using (1) and (2) the total drag-torque on the hairs is determined. This dragtorque increases approximately proportional to the hair length cubed $\left(\mathrm{L}_{0}\right)^{3}$, when $\mathrm{L}_{0}<\beta^{-1}$ showing the importance of hair-length for optimised sensitivity.

The drag-torque shows a high-pass frequency response to the free-stream velocity, with drag-torques up to $10^{-10} \mathrm{Nm}$. The total response, including the mechanical response of the sensor, shows a band-pass behaviour. The many filiform hairs on the cricket's cerci vary in their band-pass behaviour, because the mechanical properties and drag-torque are dependent on the hair length. By combining many dissimilar filiform hairs the cricket is able to create a sensitive sensory system, which has a relatively balanced frequency spectrum [9].

\section{Artificial hairs}

It is the aim of this work to learn from natural sensors as found on crickets and to produce comparable structures using MEMS technology. Several groups have worked on the realisation of artificial hairs for flow sensing [13-17]. Fabrication of hairs in the wafer plane is straightforward since surface micromachining techniques can be used [14] and hairs can be erected after fabrication. To this end $\mathrm{Li}$, et al. proposed to use the socalled plastic deformation magnetic assembly (PDMA) method $[15,16]$. In the PDMA process, a magnetic field is used to bend surface micromachined beams. The beams are plastically deformed, so that they remain bent after the magnetic field is removed. Despite the ease of fabrication surface micromachined hairs cannot easily be combined into high-density arrays.

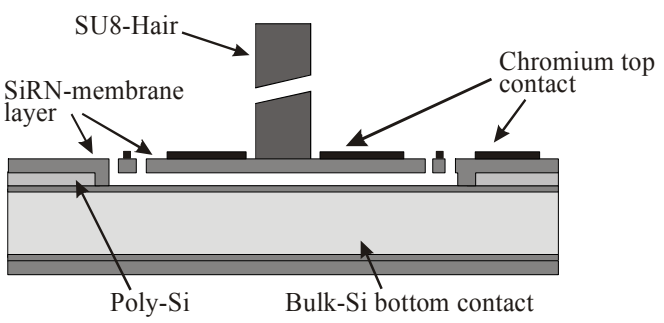

Figure 3. Sensor structure with SU-8 hair.

In this work hair-sensors are fabricated by a combination of a sacrificial poly-silicon technology, to form 
silicon-nitride suspended membranes, and SU-8 polymer processing for hair fabrication. Figure 3 shows a schematic drawing of the proposed sensor structure. A condensed fabrication scheme is shown in Figure 4.

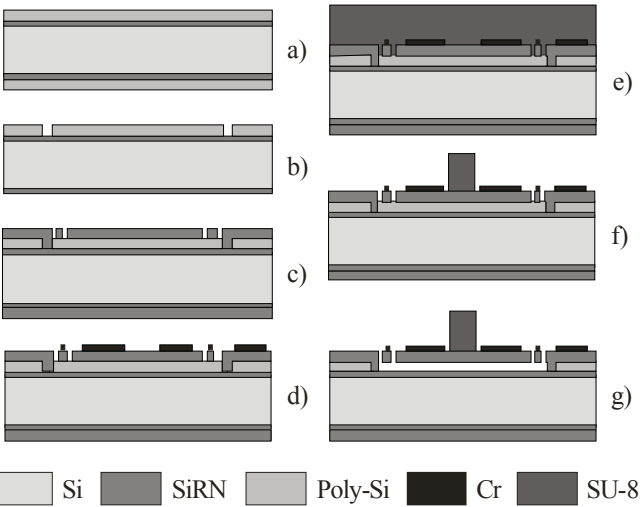

Figure 4. Condensed process flow of the sensors.

Processing starts with a highly conductive silicon wafer, since the substrate is used as a common electrode for capacitive read-out. A $100 \mathrm{~nm}$ thin silicon-nitride layer is deposited by LPCVD, for the protection of the substrate during later etching of the sacrificial layer. A $1 \mu \mathrm{m}$ sacrificial poly-silicon layer is deposited by LPCVD and patterned to form etch-stop trenches. A second, $1 \mu \mathrm{m}$ thick silicon nitride layer is deposited and patterned to form the membranes and suspension springs, followed by sputtering of a $20 \mathrm{~nm}$ chromium layer. A layer of SU-8 photo-resist is spin-coated on the wafer surface. The SU-8 resist is illuminated and developed to create artificial hairs. Processing is completed by dry etching of the sacrificial poly-silicon layer, thereby releasing the sensor structures, without affecting the SU-8 hairs (Figure 4g). Figure 5 shows an array of spiralsuspended sensory hairs with SU-8 hairs of $470 \mu \mathrm{m}$. The realisation of SU-8 hairs on top of the sensor structure was achieved with high yield in a relatively simple fabrication process.

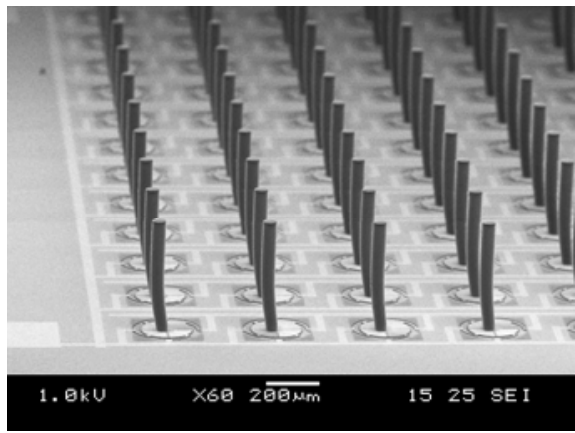

Figure 5. Array of spiral-suspended sensory hairs.

A disadvantage of SU-8 hairs is that the hair length is fixed on a given substrate by the spun SU-layer thickness, except when multiple layers and exposures of SU-8 are used. It is possible, however, to vary the diameter of the artificial hairs, which can be used alternatively to vary the amount of drag-force on the hair and the resonance frequency.

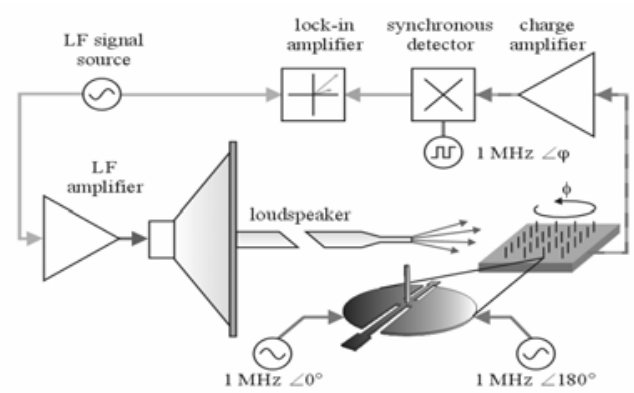

Figure 6. Acoustic flow measurement setup.

Characterisation with acoustic flows was done employing capacitive amplitude modulation of two $1 \mathrm{MHz}$ electrical signals. The electrical signals need to be taken in phase to acquire a common-mode translational signal from the sensor. A differential-mode rotational signal is acquired when the electrical signals are taken $180^{\circ}$ out of phase (figure 6). The AM signal from the sensor is amplified and demodulated, by the charge amplifier and synchronous detector. The resulting signal is fed to a lock-in amplifier, which is used to lock-in on the frequency of the LF signal source. A flow source, a loudspeaker combined with a tube, was used to determine the frequency response measured with the artificial sensory hairs, applying a differential-mode measurement. A reference particle velocity sensor [19] was used for comparison.

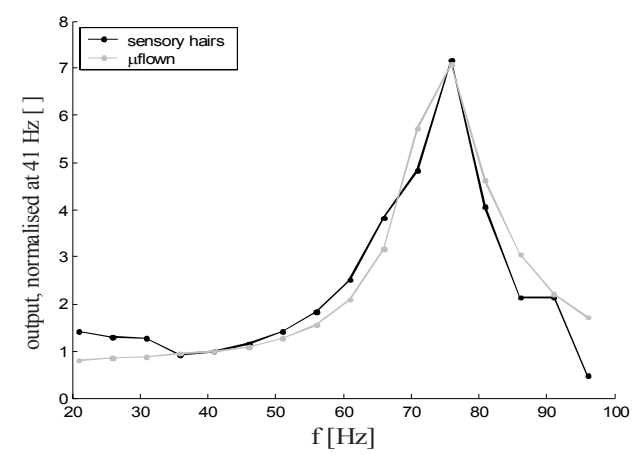

Figure 7. Frequency response of a spiral-suspended hair sensor array.

Both measurements were normalised to the amplitude obtained at $41 \mathrm{~Hz}$. Figure 7 shows that the flow source gives maximum output at $75 \mathrm{~Hz}$, as measured by both sensors whereas no clear indications are found for any resonances within the measurement range.

Directional flow-sensitivity measurements have been carried out by arrays of artificial sensory hairs using a stepper-motor for rotation, and flow amplitudes in the range $0.1-1 \mathrm{~m} \cdot \mathrm{s}^{-1}$. The lock-in amplifier measures the amplitude and phase angle for each rotation angle $\phi$ of the device (figure 6). Figure 8 shows the results of a 
differential-mode rotational measurement on an array of hair sensors. The amplitude of the output of the sensor displays a figure of eight showing that the sensor has a preferred directional sensitivity. A sharp transition can be observed in the phase at rotation angles roughly $0^{\circ}$ and $180^{\circ}$ (not shown here) where the flow direction over the electrodes changes sign.

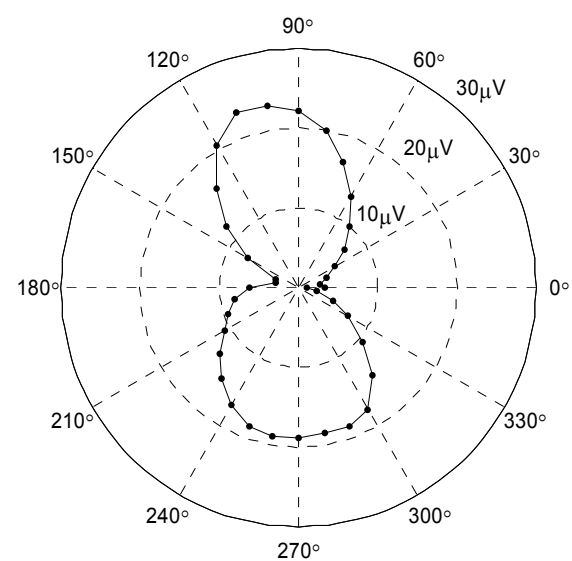

Figure 8. Directivity acoustic measurement on a spiralsuspended hair sensor array: amplitude versus $\phi$

\section{Artificial hairs as a model system}

The availability of functional hair-sensor arrays forms an invitation for research on acoustic sensing and perception. Using the many hairs in parallel various aspects of biological sensing may be mimicked:

- Using multiple spin and exposure cycles arrays of dissimilar hairs showing variability in resonance frequency may be fabricated.

- Adaptation may be obtained using biasing of the electrode structures to utilize effective stiffness weakening by electrostatic forces. This will lead to a adaptive change of resonance frequency of the hairs.

- Hairs may be fabricated using local variability to obtain localised frequency response in order to (partly) mimic artificial cochlea [1].

- Since the capacitive structures are amenable to electrostatic actuation via the electrodes one may use additional signals. Mixing of the electrostatic and acoustic signals may be utilised for parametric mixing for filtering and gain [19].

- Using available thermal and acoustic noise, or by adding mechanical noise through electrostatic actuation one may investigate stochastic concepts (stochastic resonance) on the scale of hundreds of hairs.

- Exploiting charge generation from piezo-electric material in future devices, hair-sensors may be directly integrated with living neuronal networks.

Apart from the various aspects of perception studies, the sensor structures provide interesting possibilities for distributed sensing. This allows for determination of fields (rather than single valued average quantities) e.g. the observation of micro-turbulence. Likewise, comparable arrays may be used for tactile sensing.

\section{Conclusion}

We have shown functional acoustic flow sensorarrays based on the mechano-hair sensors found on crickets. The arrays with hundreds of hairs allow for investigation of various sensing and perceptions schemes like stochastic resonance, parametric amplification, mechanical filtering, etc.

\section{Acknowledgements}

We thank: Erwin Berenschot and Meint de Boer for their processing advice, our colleagues in the EU project CICADA for stimulating discussions and input to this work and the EU for financing support through project IST 200134718 (CICADA) [20] in the Lifelike Perception Programme.

\section{References}

[1] K. Snyder et. al, F. Sachs, W. Brownell, Chapter 6 in "Sensors and Sensing in Biology and Engineering", ed. Barth, Humphry and Secomb (Springer) Vienna, 2003.

[2] T. Wellens, V. Shatokhin and A. Buchleitner, Rep. Prog. Phys. 67, 2004.

[3] J. Levin, J. Miller, Nature, Vol 380, 1996, 165-168.

[4] S. Bahar, F. Moss, Mathematical Biosciences Vol. 188, 2004, 81-97.

[5] T. Shimozawa, J. Murakami, T. Kumagai, Chapter 10 in "Sensors and Sensing in Biology and Engineering", ed. Barth, Humphry and Secomb (Springer), Vienna, 2003.

[6] F.G. Barth, Cur. Opinion in Neurobiology, Vol-14, 2004, 415-422.

[7] S. Coombs, Autonomous Robots 11, 2001, 255-261.

[8] Photo courtesy G. Jeronimidus, R. Seidel, K. Winwood, Biomimetics Centre, Reading University

[9] O. Dangles, J. Casas, Institut de Recherche en Biologie de l' Insecte, private communication

[10] Landolfa M.A. and Jacobs G.A. Journal of Comparitive Physiology A 177, 1995, 759-766

[11] T. Shimozawa, T. Kumagai, Y. Baba, Journal of Comparative Physiology A 183, 171-186

[12] J.A.C. Humphrey, et al. Philosophical Transactions: Biological Science 340, 423-444

[13] Y. Ozaki, T. Ohyama, T. Yasuda, and I. Shimoyama Proc. MEMS 2000 (Miyazaki, Japan) 531-537

[14] J. Chen, Z. Fan, J. Zou, J. Engel, and C. Liu, Journal of Aerospace Engineering 16, 85-97.

[15] J. Li, J. Chen, C. Liu, NanoSpace 2000, Houston.

[16] J. Zou, J. Chen, C. Liu, IEEE/ASME Journal of MEMS 10, 302-309.

[17] Z. Fan, J. Chen, J. Zou, D. Bullen, C. Liu, and F. Delcomyn, Journal of Micromechanics and Microengineering 12, 655-661.

[18] H-E. de Bree, et al., Sensors and Actuators A 54 552-557.

[19] Dustin W. Carr, Stephane Evoy, Lidija Sekaric, H. G. Craighead, and J. M. Parpia, Ap. Phys. Lett. 77, 1545.

[20] http://www.bionics-cicada.org/ 